Communications in Physics, Vol.21, No. 2 (2011), pp. 161-168

\title{
OPTICAL BISTABILITY EFFECT OF TWO-PORT NONLINEAR FIBER MACH-ZEHNDER INTERFEROMETER
}

\author{
HO QUANG QUY \\ Institute for Applied Physics-NEWTECHPRO \\ VU NGOC SAU \\ Vinh University \\ NGUYEN VAN HOA \\ Hong Duc University \\ NGUYEN THI THANH TAM \\ Quang Nam University
}

\begin{abstract}
In this article, the dependence of bistability characteristic of the two-port nonlinear fiber Mach-Zehnder interferometer (TPNFMZI) on initial phase shift, wavelength of input signal, nonlinear coefficient of refractive index and length of nonlinear fiber is investigated and discussed. The results of this work play an important role in designing the TPNFMZI and its application in the future.
\end{abstract}

\section{INTRODUCTION}

The optical bistability effect is a phenomenon in which two stable output states occur in proportion to one input state when a laser beam transmits through a nonlinear medium. The cause of that phenomenon is abrupt change of states of the physical system when the certain physical conditions satisfies. This is an especially important property of TPNFMZI (Fig. 1) which contributes to the creation of high-speed quantum computer system. Still now, the bistability characteristic of it, that depends on the principle parameters has not been completely investigated. Based on the results of our previous works [14], the influence of principle parameters on bistability characteristic of the TPNFMZI is simulated and discussed.

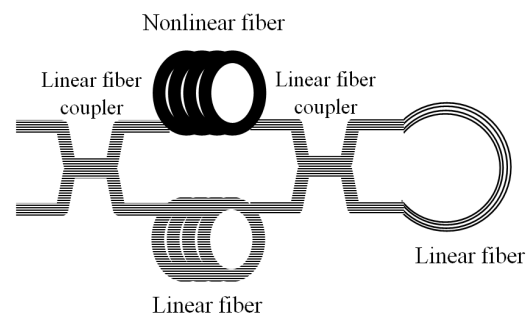

Fig. 1. The TPNFMZI. 
The expression describing the relation between output and input intensities of the TPNFMZI is as follows [14]

$$
\begin{aligned}
& I_{\text {out }}=I_{\text {in }} \times\left\{\left[-\left(2 \eta_{2}-1\right)\left(1-\eta_{1}\right)-2 \sqrt{\eta_{1} \eta_{2}\left(1-\eta_{1}\right)\left(1-\eta_{2}\right)}\right]^{2}\right. \\
& +\left[\left(2 \eta_{2}-1\right) \eta_{1}-2 \sqrt{\eta_{1} \eta_{2}\left(1-\eta_{1}\right)\left(1-\eta_{2}\right)}\right]^{2}-2\left[\left(2 \eta_{2}-1\right)\left(1-\eta_{1}\right)+2 \sqrt{\eta_{1} \eta_{2}\left(1-\eta_{1}\right)\left(1-\eta_{2}\right)}\right] \\
& \times\left[\left(2 \eta_{2}-1\right) \eta_{1}-2 \sqrt{\eta_{1} \eta_{2}\left(1-\eta_{1}\right)\left(1-\eta_{2}\right)}\right] \\
& \left.\times \cos \left(\frac{2 \pi n_{2} L}{\lambda\left(1-\eta_{1}\right)}\left[I_{\text {out }}+I_{\text {in }} \times\left(\eta_{1}\left(1-\eta_{1}\right)+4 \eta_{1} \eta_{2}\left(1-\eta_{1}\right)\left(1-\eta_{2}\right)+\eta_{1}^{2}\left(2 \eta_{2}-1\right)^{2}\right)\right]+\Delta \varphi\right)\right\}
\end{aligned}
$$

where $I_{\text {in }}, I_{\text {out }}$ are the input and output intensities, respectively; $\eta_{1}, \eta_{2}$ are the transfer efficiencies of two linear fiber coupler, respectively; $n_{2}, L$ are the nonlinear coefficient of refractive index and the length of nonlinear fiber, respectively; $\Delta \varphi$ is the initial phase shift and $\lambda$ is wavelength of the input laser beam.

\section{The dependence of the optical bistability of TPNFMZI on the parameters}

For simplicity, consider the laser beam transmits through the TPNFMZI which has two couplers with transfer efficiencies $\eta_{1}=\eta_{2}=0.5$. We can rewrite the equation (1) as follows

$$
I_{\text {out }}=I_{\text {in }}\left[\frac{1}{2}+\frac{1}{2} \cos \left\{\frac{4 \pi n_{2} L}{\lambda}\left(I_{\text {out }}+\frac{1}{2} I_{\text {in }}\right)+\Delta \varphi\right\}\right] .
$$

From the equation (2), we simulate the dependence of the optical bistability characteristic of the TPNFMZI on $\Delta \varphi, \lambda, n_{2}$ and $L$.

\section{II.1. The dependence of the optical bistability of the TPNFMZI on the initial phase shift $(\Delta \varphi)$}

Using the equation (2), the dependence of the output intensity on the input intensity with different values of $\Delta \varphi$, that changes from 0 to $2 \pi$, is simulated and shown in Fig. 2 .

Figure 2 a shows that the optical bistability effect occurs with every $\Delta \varphi$. However, there are multistability phenomena when input intensity is high enough. For example, $I_{\text {in }}>I_{\text {in } 2}$, the multistability phenomena occurred in Fig. 2b. There are some important values to describe the bistability characteristic of TPNFMZI as, $I_{i n 1}, I_{i n 2}$ are bistability thresholds, $I_{\text {inp }}, I_{\text {outp }}$ are positions of point $\mathrm{P}, \Delta I_{\text {in }}$ is the width of the bistability effect and $\Delta I_{\text {out } 1}, \Delta I_{\text {out } 2}$ are the output intensity ranges in which $I_{\text {out } 1}, I_{\text {out } 2}$ change abruptly (see Fig. 2b).

To see more clearly the dependence on $\Delta \varphi$ of the bistability characteristic, we divide $\Delta \varphi$ into parts corresponding to the suitable input intensity ranges so that the bistability effect appears as shown in Fig. 3.

Fig. 3a, b show that with increasing of $I_{\text {in }}, I_{\text {out }}$ increases to the first maximum (correlating to point $\mathrm{P}\left(I_{\text {inp }}, I_{\text {outp }}\right)$ in Fig. $\left.2 \mathrm{~b}\right)$ and then $I_{\text {out }}$ decreases, when $I_{\text {in }}=I_{\text {in } 2}$ the bistability effect occurs as presented above. The values such $I_{i n 1}, I_{i n 2}, \Delta I_{\text {in }}, \Delta I_{\text {out } 2}$, 


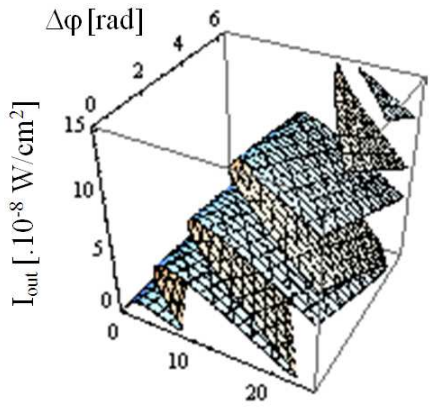

a $\quad \mathrm{I}_{\text {in }}\left[\cdot 10^{-8} \mathrm{~W} / \mathrm{cm}^{2}\right]$

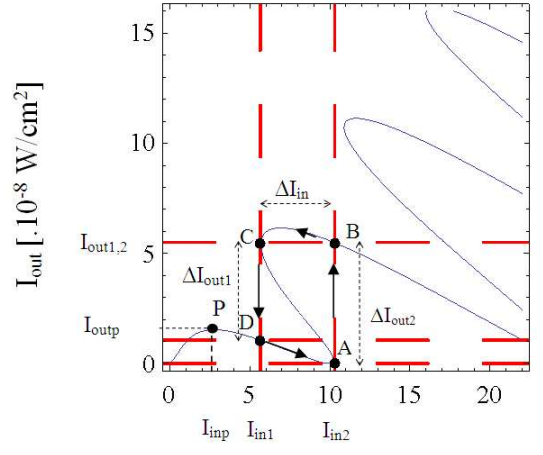

b $\quad \mathrm{I}_{\text {in }}\left[\cdot 10^{-8} \mathrm{~W} / \mathrm{cm}^{2}\right]$

Fig. 2. (a) The series of the output-input intensity relation curves of the TPNFMZI when $\Delta \varphi$ changes from 0 to $2 \pi$, (b) The output-input intensity relation curve of the TPNFMZI when $\Delta \varphi=-0.3 \pi$, with $L=10(\mathrm{~cm}), n_{2}=$ $10^{-14}\left(\mathrm{~cm}^{2} / W\right), \lambda=1.53 \mu m$.
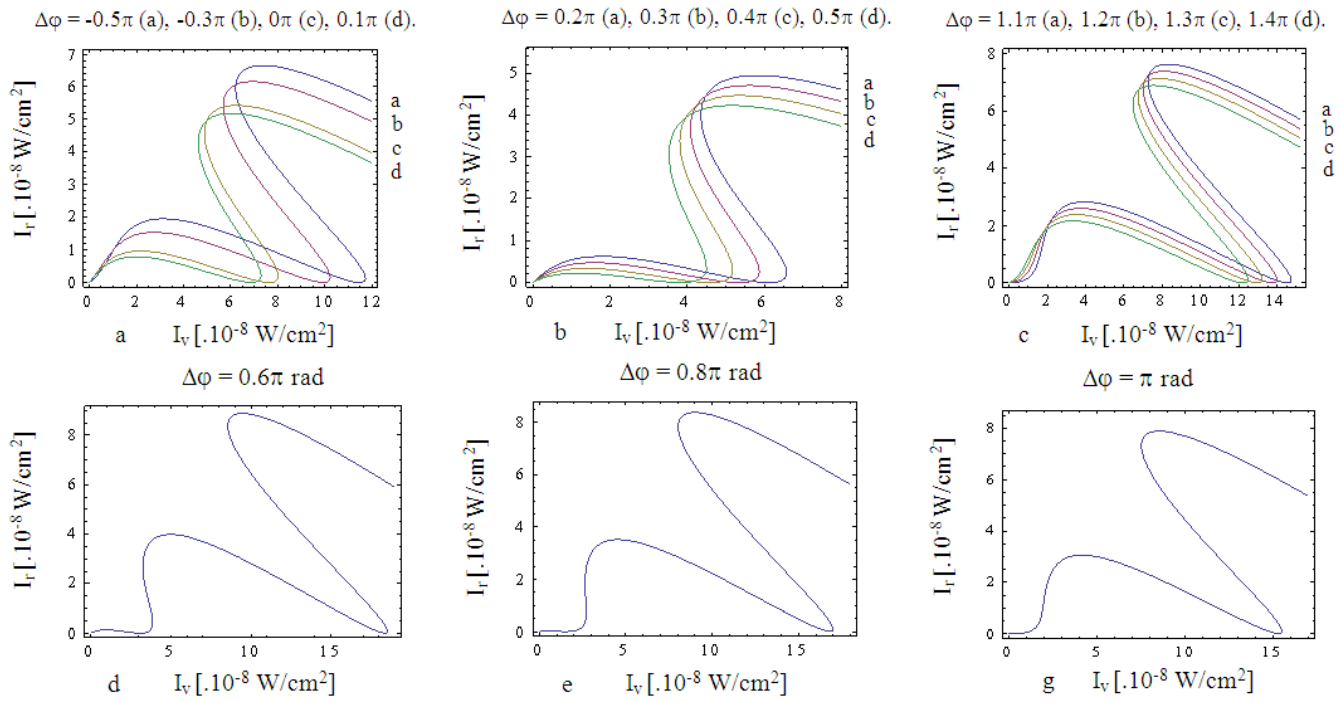

Fig. 3. The series of the bistability characteristic curves of the TPNFMZI when change $\Delta \varphi$, (a) $\Delta \varphi=-0.5 \pi,-0.3 \pi, 0,0.1 \pi$ corresponding to a, b, c, d curves, respectively, (b) $\Delta \varphi=0.2 \pi, 0.3 \pi, 0.4 \pi, 0.5 \pi$ corresponding to a, b, c, d curves, respectively, (c) $\Delta \varphi=1.1 \pi, 1.2 \pi, 1.3 \pi, 1.4 \pi$ corresponding to a, b, c, d curves, respectively, (d, e, g) the bistability characteristic curves corresponding to $\Delta \varphi=$ $0.6,0.8 \pi, \pi$, respectively.

$\Delta I_{\text {out } 1}, I_{\text {outp }}$ and declination of the bistability characteristic curve in Fig. 3a is larger than that in the Fig. $3 \mathrm{~b}$.

From figures $3 \mathrm{c}, \mathrm{d}, \mathrm{e}, \mathrm{g}$, there is a little change, at first $I_{\text {out }}$ is almost unchanged or changed very little $\left(I_{\text {out }} \approx 0\right)$ with increasing of $I_{\text {in }}$ and then $I_{\text {out }}$ also increases. Especially, 
in Fig. 3e when $I_{\text {in }}$ increases to $I_{\text {inp }}, I_{\text {out }}$ increases abruptly to $I_{\text {outp }}$, then process occurs similarly as in the figures $3 \mathrm{a}$, b. The values such $I_{\text {in } 1}, I_{\text {in } 2}, \Delta I_{\text {in }}, \Delta I_{\text {out } 2}, \Delta I_{\text {out } 1}, I_{\text {outp }}$ and declination of these bistability characteristic curves is larger than that in the Fig. 3a.

Depending on purpose of the use we can choose suitable value of phase shift. For example, if we want $I_{\text {outp }}$ to be high and $\Delta I_{\text {in }}$ to be large, should design TPNFMZI in such way that signals going through it have the initial phase shift in $0.5 \pi<\Delta \varphi<2 \pi$.

From Fig. 3 we see $I_{\text {out } 2} \approx I_{\text {out } 1}$ only when $\Delta \varphi=-0.3 \pi$. Therefore, in order to take advantage of investigation of the dependence of bistability characteristic on other parameters we choose $\Delta \varphi=-0.3 \pi$ (see Fig. 4).
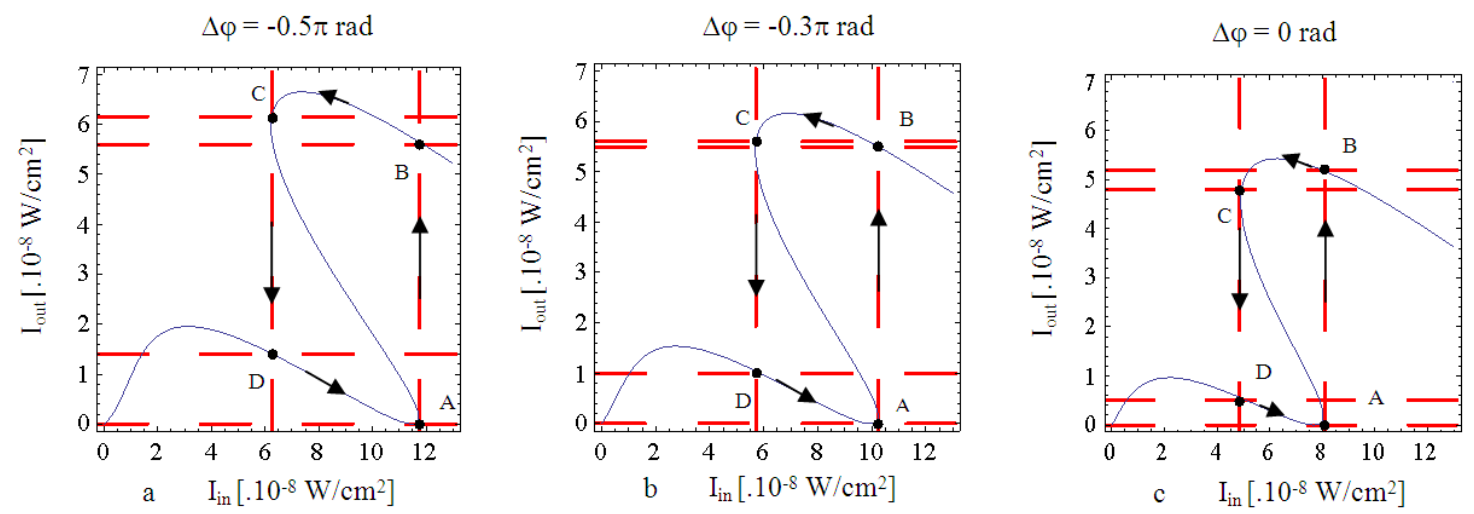

Fig. 4. The series of the bistability characteristic curves of the TPNFMZI when change $\Delta \varphi$ (a) $\Delta \varphi=-0.5 \pi$, (b) $\Delta \varphi=-0.3 \pi$, (c) $\Delta \varphi=0$.

\section{II.2. The dependence of the optical bistability of the TPNFMZI on the wave- length $(\lambda)$ of input signal}

In optical communications, the part of the used infrared spectrum is divided into six bands based on availability of light sources transmitting/absorbing materials (fibers) and detectors (see Table 1) [11]

Table 1. Infrared bands used in optical communications

\begin{tabular}{|c|l|c|}
\hline Band & \multicolumn{1}{|c|}{ Descriptor } & Wavelength range(nm) \\
\hline O & band Original & $1260-1360$ \\
E & band Extended & $1360-1460$ \\
S & band Short wavelength & $1460-1530$ \\
C & band Conventional & $1530-1565$ \\
L & band Long wavelength & $1565-1625$ \\
U & band Ultralong wavelength & $1625-1675$ \\
\hline
\end{tabular}

The C-band is the dominant band for long-distance telecommunication networks.

The bistability characteristic of TPNFMZI with different values of wavelength of input signal, that changes from $1260 \mathrm{~nm}$ to $1675 \mathrm{~nm}$, is simulated and shown in Fig. 5 


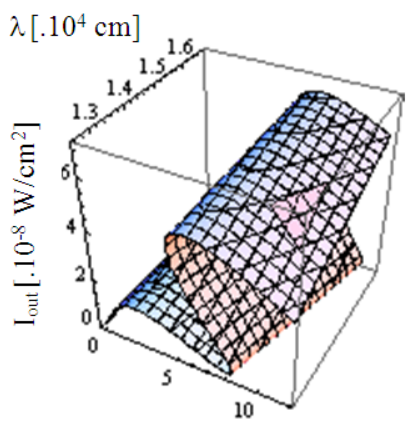

a $\mathrm{I}_{\text {in }}\left[\cdot 10^{-8} \mathrm{~W} / \mathrm{cm}^{2}\right]$
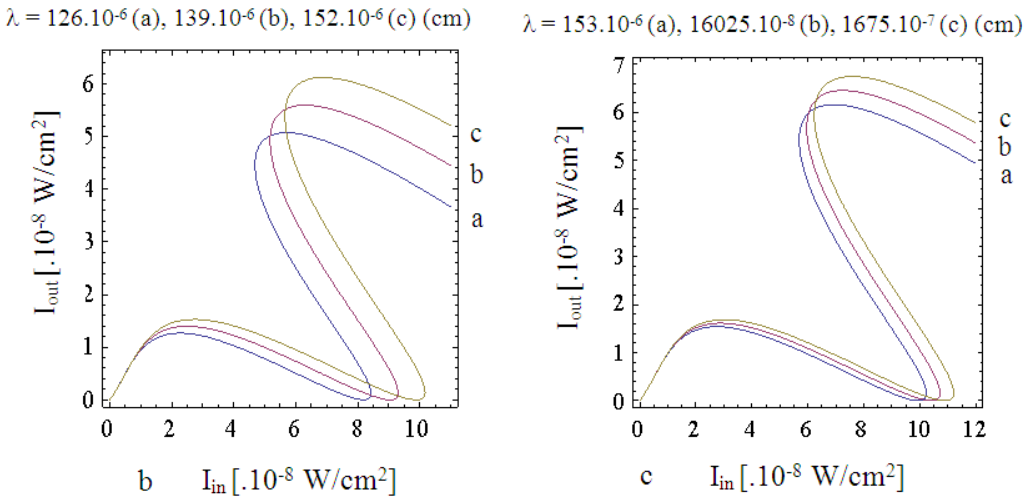

Fig. 5. The series of the output-input intensity relation curves of the TPNFMZI when $\lambda$ changes from $1260 \mathrm{~nm}$ to $1675 \mathrm{~nm}$, with $L=10(\mathrm{~cm}), n_{2}=10^{-14}\left(\mathrm{~cm}^{2} / W\right)$ and $\Delta \varphi=-0.3 \pi$, (a) $\lambda=1260-1675 \mathrm{~nm}$, (b) $\lambda=1260 \mathrm{~nm}, \lambda=1390 \mathrm{~nm}$, $\lambda=1520 \mathrm{~nm}$ corresponding to a, b, c curves, respectively, (c) $\lambda=1530 \mathrm{~nm}$, $\lambda=1602.5 \mathrm{~nm}, \lambda=1675 \mathrm{~nm}$ corresponding to a, b, c curves, respectively.

From Fig. 5a we see when $\lambda$ changes from $1260 \mathrm{~nm}$ to $1675 \mathrm{~nm}$, the optical bistability effect occurs. However, there are multistable phenomena which have been appeared in the Fig. 5a because of input intensity is too high, for example when $I_{i n}>I_{i n 2}$ the multistability phenomena will appear.

To see more clearly the dependence on $\lambda$ of the bistability characteristic, we divide wavelength range into parts corresponding to the suitable input intensity ranges so that the bistability effect appears as Fig. $5 \mathrm{~b}$ and $5 \mathrm{c}$. It is easy to see when $\lambda$ increases the values such $I_{\text {in } 1}, I_{\text {in } 2}, \Delta I_{\text {in }}, \Delta I_{\text {out } 2}, \Delta I_{\text {out } 1}$ increase and $I_{\text {out } 2}=I_{\text {out } 1}$.

From International Telecommunication Union (ITU), the C-band is the dominant band for long-distance telecommunication networks and it is hardly absorbed when transmitted optical fiber. Therefore, we have used input signals which have wavelength changes from 1530 to $1565 \mathrm{~nm}$ to simulate (see Fig. 6).

Fig. 6 shows that when $I_{\text {in }}$ increases the values such $I_{\text {in } 1}, I_{\text {in } 2}, \Delta I_{\text {in }}, \Delta I_{\text {out } 2}, \Delta I_{\text {out } 1}$, $I_{\text {outp }}$ increase linearly and $I_{\text {out } 2}=I_{\text {out } 1}$. When $\lambda=1530 \mathrm{~nm}$, the bistability threshold is the smallest. Therefore, we have used $\lambda=1530 \mathrm{~nm}$ to simulate in the next investigation.

\section{II.3. The dependence of the optical bistability of the TPNFMZI on the non- linear coefficient of refractive index $\left(n_{2}\right)$}

The dependence of the output intensity on the input intensity with different values of $n_{2}$, that changes from $10^{-15}$ to $10^{-13}\left(\mathrm{~cm}^{2} / W\right)$, is simulated and shown in Fig. 7 .

From Fig. 7, we can see that when $n_{2}$ increases, the values such $I_{i n 1}, I_{i n 2}, \Delta I_{i n}$, $\Delta I_{\text {out } 2}, \Delta I_{\text {out } 1}, I_{\text {outp }}$ recrease linearly and $I_{\text {out } 2}=I_{\text {out } 1}$. These show that when nonlinear coefficient of refractive index is changed, the output-input intensity relation changes, but the shape of the optical bistability characteristic curves still does not change. When we change the optical fiber medium with increasing nonlinear coefficient of refractive index 

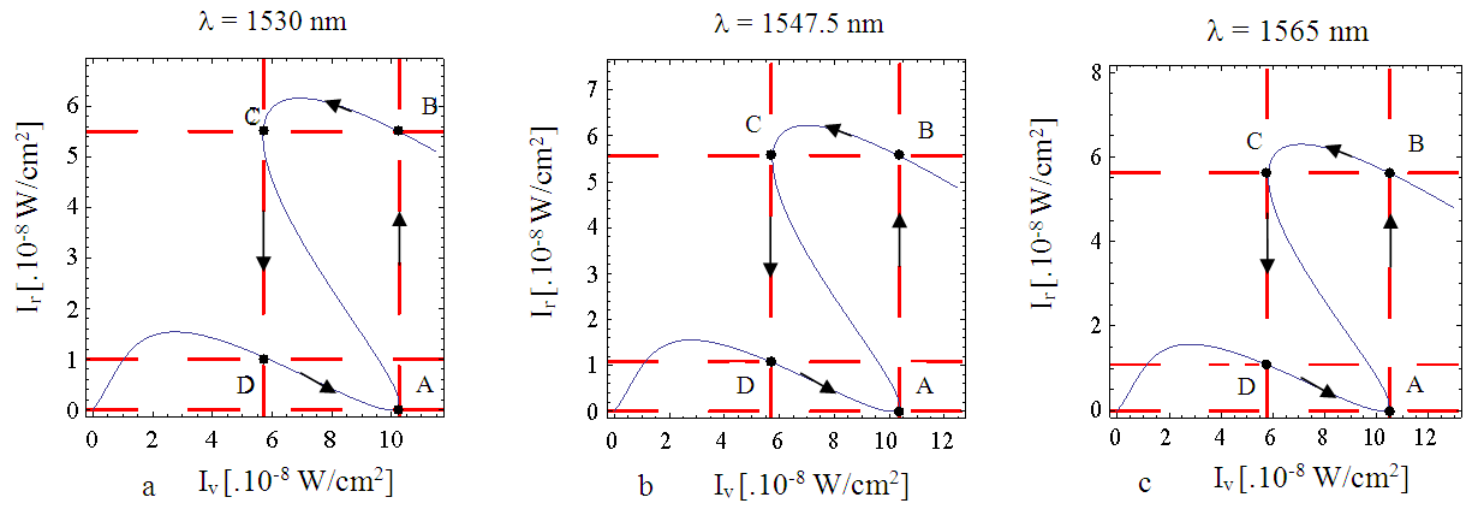

Fig. 6. The stability characteristic curves of the TPNFMZI when $\lambda$ changes from $1530 \mathrm{~nm}$ to $1565 \mathrm{~nm}$, (a) $\lambda=1530 \mathrm{~nm}$, (b) $\lambda=1547.5 \mathrm{~nm}$, (c) $\lambda=1565 \mathrm{~nm}$.
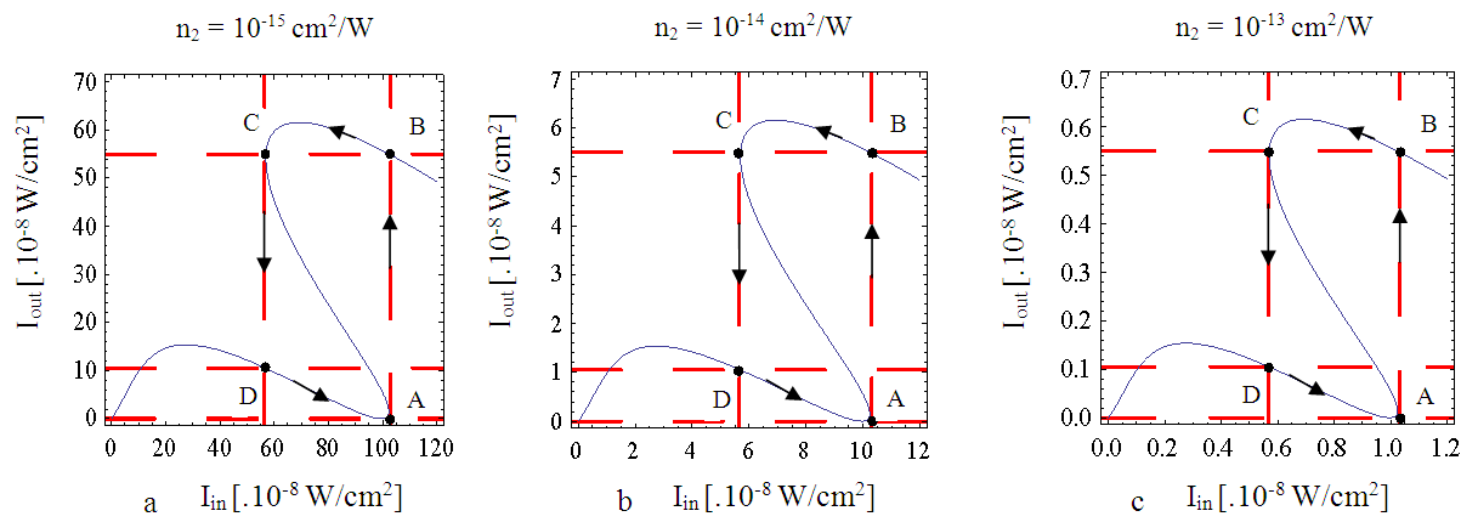

Fig. 7. The optical bistability characteristic curves of the TPNFMZI in proportion to each nonlinear coefficient of refractive index value, with $L=10(\mathrm{~cm}), \Delta \varphi$ $=-0.3 \pi$ and $\lambda=1.53 \mu \mathrm{m}$, (a) $n_{2}=10^{-15}\left(\mathrm{~cm}^{2} / W\right)$, (b) $n_{2}=10^{-14}\left(\mathrm{~cm}^{2} / W\right)$, (c) $n_{2}=10^{-13}\left(\mathrm{~cm}^{2} / W\right)$

$n_{2}$ to 10 times, in contrast the output-input intensity relation decreases 10 times, but the optical bistability characteristic curve keeps its shape. This result helps to choose the suitable $n_{2}$ value so that there is the bistability threshold needed in TPNFMZI application.

\section{II.4. The dependence of the optical bistability of the TPNFMZI on the non- linear fiber length $(L)$}

The dependence output intensity on input intensity with different values of the nonlinear fiber length $L$, that changes from $1 \mathrm{~cm}$ to $100 \mathrm{~cm}$, is simulated and shown in Fig. 7.

Fig. 8 shows that when $L$ increases to 10 times, in contrast the output-input intensity relation decreases 10 times, but the optical bistability characteristic curve keeps its shape. 

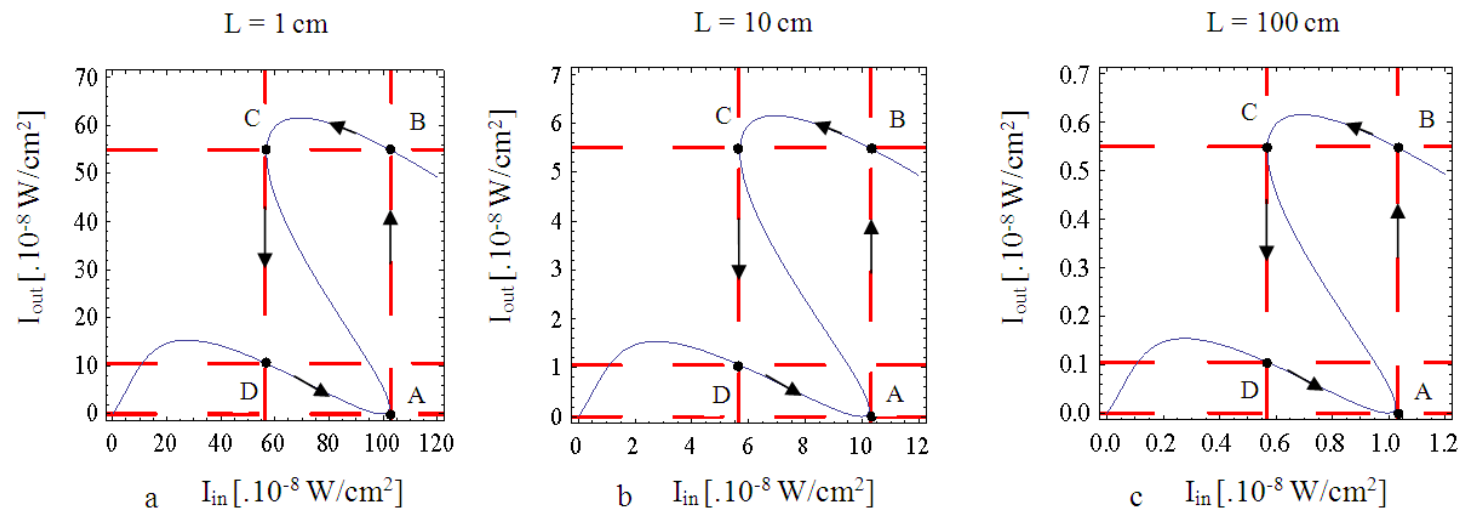

Fig. 8. The optical bistability characteristic curve of the TPNFMZI in proportion to each optical nonlinear fiber length value, with $\Delta \varphi=-0.3 \pi, \lambda=1.53 \mu \mathrm{m}$ and $n_{2}=10^{-14}\left(\mathrm{~cm}^{2} / \mathrm{W}\right)$, (a) $L=1 \mathrm{~cm}$, (b) $L=10 \mathrm{~cm}$, (c) $L=100 \mathrm{~cm}$

\section{CONCLUSIONS}

Based on the expression describing the output-input intensity relation of the TPNFMZI, the dependence of the bistability characteristic on the principle parameters is simulated using the Mathematica software. The bistability characteristic of TPNFMZI depends not only on input intensity, but also on initial phase shift, wavelength of the input signal, nonlinear coefficient of refractive index and length of nonlinear fiber. The optical bistability effect occurs with every $\Delta \varphi, \lambda, n_{2}$ and $L$ in each suitable input intensity range. When the principle parameters change, the shape of the bistability characteristic curves change and splited into three shape groups. The first group, that with increasing of $I_{\text {in }}, I_{\text {out }}$ increases to point $\mathrm{P}\left(I_{\text {inp }}, I_{\text {outp }}\right)$ and then $I_{\text {out }}$ decreases and finally the bistability effect occurs. The second group, that with increasing of $I_{\text {in }}, I_{\text {out }}$ almost does not change $\left(I_{\text {out }} \approx 0\right)$, then the bistability effect occurs. The third group, that with increasing of $I_{\text {in }}, I_{\text {out }}$ changes into multi-declination, then the bistability effect occurs but not clear. Especially, when the nonlinear fiber length or nonlinear refractive index increase to 10 times, the output-input intensity relation decreases 10 times, but the optical bistability characteristic curve keeps its shape. The results leads to use the TPNFMZI for reshaping of optical signal in the next article.

\section{REFERENCES}

[1] M. C. D. Andrew, Opt. Switching, OPN (2005) 34.

[2] L. Brzozowski et al., J. of Lightwave Technology 19 (2001) 114-119.

[3] M. Cai et al, Opt. Let. 25 (2000) 1430.

[4] W. Demtroder, Laser spectroscopy, (1982).

[5] A. Erlacher et al., J. of Appl.Phys 95 (2004) 2927-2929.

[6] N. V. Hoa, H. Q. Quy, V. N. Sau, Commun. in Phys. 15 (2005) 223-228.

[7] N. V. Hoa, H. Q. Quy, D. X. Khoa, Proc. of GVS6, Chemnitz (2003) 144-147.

[8] J. M. Jonathan, Summer School, Doson (2004) 245.

[9] H. T. Martin et al, Otp. Tech. Letter, 31 (2001) 411-415. 
[10] H. Q. Quy, V. N. Sau, N. V. Hoa, Commun. in Phys. 13 (2003) 157-164.

[11] Ramaswami, Rajiv, Optical Fiber Communication, IEEE, (2002).

[12] H. Sakata, Appl. Phys. 40 (2001) 240-248.

[13] M. Soljacic et al, Phys. Rev. E66 (2002) 05560-1-05560-4(R).

[14] H. Q. Quy, N. T. T. Tam and N. V. Hoa, Comm. in Phys. 20 (2010) 371-376.

Received 13 August 2010. 